\title{
Fingerprint Image Compression using Sparse Representation and Enhancement with Wiener2 Filter
}

\author{
Divya Joseph \\ Amal Jyothi College of Engineering \\ Kottayam, Kerala, India
}

\author{
Niya Joseph \\ Amal Jyothi College of Engineering \\ Kottayam, Kerala, India
}

\begin{abstract}
A technique for enhancing decompressed fingerprint image using Wiener2 filter is proposed. First compression is done by sparse representation. Compression of fingerprint is necessary for reducing the memory consumption and efficient transfer of fingerprint images. This is very essential for the application which includes access control and forensics. So the fingerprint image is compressed using sparse representation. In this technique, first dictionary is constructed for patches of fingerprint images. Then a fingerprint is selected and the coefficients are obtained and encoded. Thus the compressed fingerprint is obtained. But when the fingerprint is reconstructed, it is affected by noise. So Wiener2 filter is used to filter the noise in the image. The ridge and bifurcation count is extracted from decompressed and enhanced fingerprints. The experiment result shows that the enhanced fingerprint image preserves more bifurcation than decompressed fingerprint image. The future analysis can be considered for preserving ridges.
\end{abstract}

Keywords: Fingerprint Compression; Sparse Representation; Enhancement; Wiener2 Filter; Minutiae Extraction

\section{INTRODUCTION}

A fingerprint is defined as a mark left by the minutiae of a human finger. Collecting of fingerprints from a crime detection scene is an major method of forensic science. Fingerprint detection refers to the automatic way of finding a match between two human fingerprints. The Federal Bureau of Investigation (FBI) deal with a massive collection of fingerprint cards which contains huge number of cards and is growing at the rate of 30,000-50,000 cards per day [1]. The FBI is digitizing these cards for storage, renewal, and transference. Because of data storage needs and the time needed to transfer a fingerprint card over a modem, these files must be compressed. Fingerprint compression is gaining much attention with the recent widespread transmission of images.

Generally, compression techniques can be classified into lossless compression and lossy compression. Lossless compression allows the original images to be recovered from the compressed data. Lossless compression techniques are used when it is important that the authentic and the decompressed images are similar. Avoiding distortion limits the efficiency of image compression. In image compression slight distortion is acceptable. So lossless compression technologies are usually employed in the outputs of lossy compression. There are several compression techniques available for image compression. Compared with general images, the fingerprint images have simpler format. Fingerprint images only contain ridges and valleys. So compression algorithms are developed by targeting fingerprint images.

\section{RELATED WORKS}

The development of image compression began with several systems from years ago. But a standard image compression method was needed to enable interoperability of equipment from different manufacturer. So JPEG [2] evolved as the first international digital image compression standard used for continuous-tone images (gray scale or color). The JPEG compression has numerous points of interest, for example, effortlessness, comprehensiveness and accessibility. However, performance is not good at lower bit-rates because of the underlying DCT scheme which is block-based. So in 1995, the JPEG-committee started to build up another wavelet-based compression standard for still pictures, to be specific JPEG 2000[3]. When compared to JPEG, JPEG 2000 has many features that support scalable and intuitive access to huge estimated picture.

The above algorithms are for compressing regular images. Focusing on fingerprint images, there are certain compression algorithms. Hopper proposed the most common fingerprint image compression algorithm called as Wavelet Scalar Quantization (WSQ) [4]. It is used by the FBI (Federal Bureau of Investigation) for the compression of $500 \mathrm{dpi}$ fingerprint images. However due to the drawback of quantizer in this method, it cannot cope with fuzzy images. Thus an enhanced WSQ fingerprint image compression algorithm is made by Tang et al [5]. A generic fingerprint image compression technique depend on wave atoms decomposition was proposed by Mohammed et al [6] which is better than the standard method for FBI fingerprint image compression, the wavelet scalar quantization (WSQ). The proposed compression scheme is based upon linear vector quantization of dissolved wave atoms illustration of fingerprints. Then coefficients which are quantized are encoded with arithmetic entropy scheme. Application of wave atoms based decomposition for fingerprint image compression results into a significant improvement in peak signal to noise ratio (PSNR) compared to FBI's WSQ fingerprint compression standard. However, wave atoms cannot deal with the fact that the edges found in fingerprints are sharp curves. This problem is solved by transforms known as contourlets[7], preserves edges.

The contourlet transform is an extension of wavelet transform in 2D using directional filter banks. The requirement of storage in multistage vector quantization (MSVQ) is lesser when compared with full search vector quantization. Multistage vector quantization is used for quantizing coefficients of contourlet transform. Huffman coding is used to encode quantized coefficients. As a representation of directional multiresolution image, the contourlet transform 
can surely grab curved and aligned geometrical formats in images. However, the contourlet transform has the shortcoming of a repetition in its oversampling ratio. Zhang and Moloney have found a nonredundant version of the contourlet transform, known as the nonredundant contourlet transform (NRCT) [8] and have demonstrated that this redundancy can be eliminated by the NRCT. With the advantages of sampling and reconstruction, the NRCT can be good enough for tracing and well coding of oriented structure in images, such as the structure of ridges in fingerprint images. Moreover, as an extension of the wavelet transform, the NRCT is easily adaptable with the wavelet transform. A new transform which associate the NRCT with the wavelet transform is called the hybrid-NRCT.

The main idea behind this paper is to compress the fingerprint based on sparse representation and later enhancement is done using Wiener2 filter. The sparse representation is efficient to preserve the minutiae contents which are required for fingerprint applications. However it is suffered by noise when decompressed and the accuracy is very low. In order to avoid that Wiener2 filter is used for removing noise from decompressed fingerprints.

The rest of our paper is organized as follows. The main principles of the techniques mentioned in the proposed method are introduced in the next section. Section 4 sums up the performance analysis of our scheme in terms of number of ridges and bifurcation count on different fingerprint images. Conclusions are provided in Section 5.

\section{PROPOSED SYSTEM}

The methods we propose consist of construction of dictionary, compression of fingerprint, its decompression and enhancement. Finally the minutiae are extracted from both the decompressed and enhanced fingerprint images.

\subsection{Construction of Dictionary}

First construction of training set is done. Then, from the training set the dictionary is obtained. For constructing the training set, select all the fingerprint images from the database and slash them into square patches of fixed size. When we get the patches after initial screening, the training samples can be constructed by applying a greedy algorithm.

- The dictionary is initially empty and the first patch is added to it.

- Then the similarity between this patch and the next patch is tested. If they are sufficiently similar, the other patch is tested; if not, the patch is appended into the dictionary. Here, the optimization problem is solved to calculate the similarity measure between two patches (eq.1).

$$
\mathrm{S}(\mathrm{P} 1, \mathrm{P} 2)=\min \|\mathrm{P} 1 /\| \mathrm{P} 1\|2 \mathrm{~F}-\mathrm{t} * \mathrm{P} 1 /\| \mathrm{P} 1 \| 2 \mathrm{~F}
$$

where $\|\cdot\| 2 \mathrm{~F}$ is the Frobenius norm. $\mathrm{P} 1$ and $\mathrm{P} 2$ are the matrices that are correspondent of two patches. $\mathrm{t}$, a parameter of the optimization problem (eq.1), is a scaling factor.

- $\quad$ Repeat the 2nd step and make sure that all patches have been tested.
Before constructing the dictionary, calculate the mean value of each patch and subtract from the corresponding patch. Construction of the dictionary is done by using a training method called K-SVD [9], [10]. An optimization problem is iteratively solved to obtain the dictionary (eq.2). $\mathrm{Y}$ is consisted of the training patches, $\mathrm{A}$ is the dictionary, $\mathrm{X}$ represents the coefficients and $\mathrm{Xi}$ is the ith column of $\mathrm{X}$. MP method [11] is used to compute the coefficients matrix $X$ in the sparse solving stage. It guarantees that the coefficient vector $\mathrm{Xi}$ has non-zero elements which is limited to $\mathrm{T}$. Then, singular value decomposition (SVD) is used to update each element in the dictionary.

$$
\text { Min A,X }\|\mathrm{Y}-\mathrm{AX}\| 2 \mathrm{~F} \text { s.t for all } \mathrm{i},\|\mathrm{Xi}\| 0<\mathrm{T}
$$

\subsection{Compression of Fingerprint}

We selected a new fingerprint for compression, cut it into square patches. It has the same size with the training patches. The size of the patches has a affects the compression efficiency. The algorithm becomes more efficient as the size increases. However, there can be rapid increase in computation complexity and the dictionary size. In order to fit the patches in the dictionary, calculate the mean of each patch and subtract from the patch. Then 10 problem is solved to compute the sparse representation for each patch. If the values of coefficients are less than a given threshold, it is treated as zero [12].

Record four kinds of information for each patch. They are the mean value, number of atoms to use the coefficients and their locations. Arithmetic encoding of the mean value, number of atoms, coefficients and their location is done and compressed value is obtained.

\subsection{Decompression of Fingerprint}

The values obtained after compression is given to the arithmetic decoder. Thus the mean value, number about how many atoms to use, the coefficients and their locations of patches are regenerated. The patches are converted into images and the decompressed image is obtained.

\subsection{Enhancement of Fingerprint}

Wiener2 filter is applied to the decompressed image to remove the noise and to preserve more minutiae content. A class of filters, referred to as Wiener filters, use interrelationship data between signal and noise to enhance image or reduce distortion. Wiener2 is a $2 \mathrm{D}$ adaptive noise removal filtering. Wiener2 lowpass fiters filter an image which is vulgarized by additive noise. Wiener 2 uses a pixel based Wiener method based on data collected from each pixel. Wiener2 filter is thus used to remove the noise from the image and thus preserve more minutiae than decompressed images.

\subsection{Minutiae Extraction}

Minutiae Extraction is applied to decompressed image and enhanced image. The image is converted to binary image. This process consists of changing the gray image into binary image, i.e, the intensity has two values: black is representing the minutiae, and white is representing the valleys and the background. A method to binarize is to use a global threshold value; however, it is not suitable for images with noise content, a more flexible method that uses mask which is rectangular in shape, and rotate it according to the direction of the ridges. 


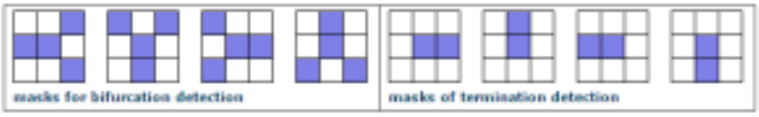

Figure 1. Pattern Masks

After the image is binarized, thinning is applied on to the image. The thinning process is to find the minutiae of one pixel width. The process consists of carrying out consecutive destruction until a set of joined lines of unit width is attained. These lines are also called skeletons. One of the characteristics of thinning is the preservation of the association and topology which can lead to creation of bifurcation artifacts and detection of false minutiae. From the binary thinned image, the minutiae are founded by using $3 * 3$ pattern masks. Samples of masks used for identifying the ridge ending and bifurcations point are shown in the Figure 1.

\section{EXPERIMENTAL ANALYSIS}

In this section, we present experiments on fingerprint database for fingerprint compression, decompression and its enhancement. Minutiae are extracted from both the decompression and its enhancement. Minutiae are extracted from both the decompressed fingerprint and its enhanced fingerprint. Then the minutiae content is analyzed in both the compressed and its enhanced fingerprint. Finally, through the experiment it is shown that the minutiae content of decompressed fingerprint. The implementation is done in MATLAB

\subsection{Databases}

To construct a dictionary of fingerprint patches, we used a set of training fingerprints. The fingerprint images can be downloaded from [13]. The size of the fingerprints in the database is $640 * 640$. The quality of fingerprints in the database is good.

\subsection{Implementation}

First the fingerprint images in the database are sliced into square patches. The dictionary is obtained from the patches by solving the optimization problem, which is described in previous section. The obtained dictionary of the images in the database is shown in Figure 2. A new fingerprint is selected and cut into square patches which have the same size with the training patches. The size of the patches has a great effect on the efficiency of compression.

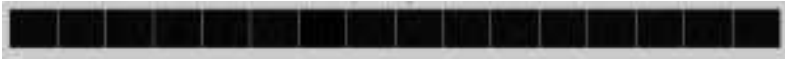

Figure 2. Dictionary of Fingerprint

The patches should be fit in the dictionary. For that, the mean of each patch needs to be calculated and subtracted from the patch. After that compute the sparse representation for each patch by solving 10 problem. Those coefficients whose absolute values are less than a given threshold are treated as zero.

For each patch, four kinds of information need to be recorded. They are the mean value, the number about how many atoms to use, the coefficients and their locations. Arithmetic encoding of the mean value, number of atoms, coefficients and their location is done and compressed value is obtained. The compression ratio is calculated and obtained as $16 \%$. When the compression ratio is too high, the minutiae contents cannot be preserved and when the compression ratio is too low, the data is not well compressed. So a moderate compression ration should be maintained.

The values obtained after compression is given to the arithmetic decoder. Thus the mean value, number about how many atoms to use, the coefficients and their locations of patches are regenerated. The patches are converted into images and the decompressed image is obtained. The obtained decompressed image is shown in Figure 3 (a). Wiener2 filter is applied to the decompressed image to remove the noise and to preserve more minutiae content. A class of filters, referred to as Wiener filters, exploit correlation information between signal and noise to enhance image or reduce distortion. Wiener2 is a 2D adaptive noise removal filtering. The obtained image is shown in Figure 4(a). The output image after enhancement is usually blurred visually. But it contains more minutiae content.

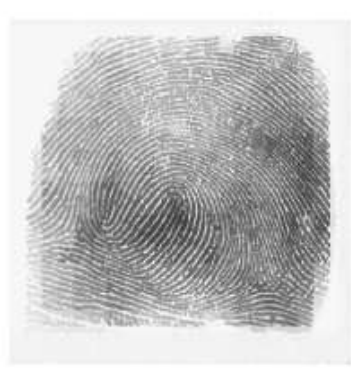

(a)

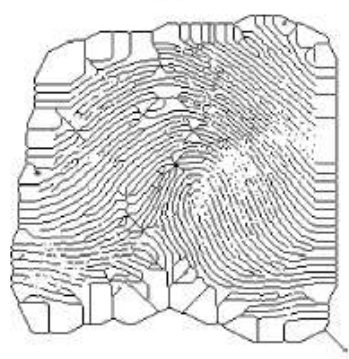

(c)

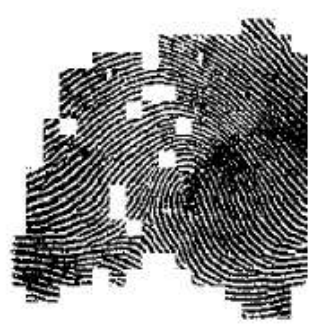

(b)

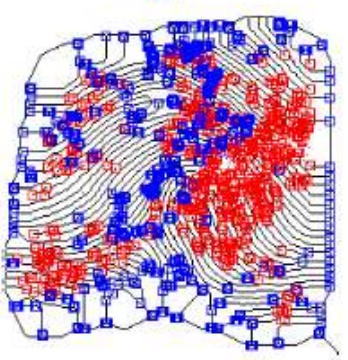

(d)
Figure 3. (a) Decompressed Fingerprint, (b) its binarization, (c) thinning and (d) Minutiae extraction.

Minutiae Extraction is applied to decompressed image and enhanced images. The image is converted to binary image. This process consist in converting the gray scale into binary image, i.e, the intensity of the image has only two values: black, representing the ridges, and white, representing the valleys and the background. A simple method to binarize is to use a global threshold value; however, it is not suitable for noisy images. So a more robust method consist of using some rectangular mask, rotate according the orientation of the ridges. The binarized images of decompressed and enhanced fingerprint are shown in Figure 3(b) and 4 (b). 


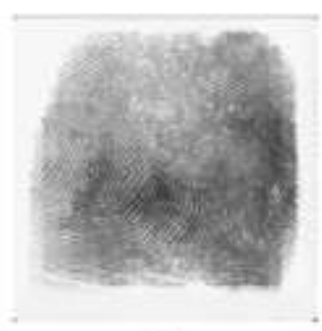

(a)

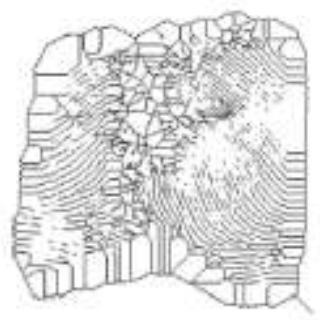

(c)

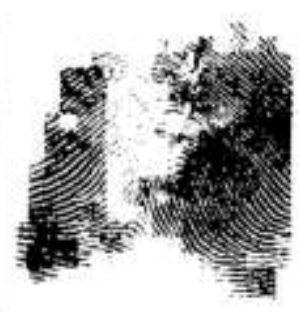

(b)

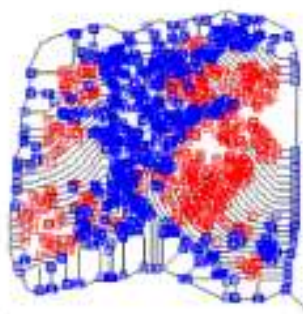

(d)
Figure 4. (a) Enhanced Fingerprint, (b) its binarization, (c) thinning and (d) Minutiae extraction.

After the image is binarized, thinning is applied on to the image. The objective of thinning is to find the ridges of one pixel width. The process consists in performing consecutive destruction until a set of connected lines of unit-width is reached. These lines are also called skeletons. Thinned images of decompressed and enhanced fingerprints are shown in Figure 3(c) and 4(c). An important property of thinning is the preservation of the association and topology which lead to creation of small bifurcation artifacts and detection of false minutiae. From the binary thinned image, the minutia is detected by using $3 * 3$ pattern masks. The minutiae extracted from decompressed and enhanced fingerprint is shown in Figure 3(d) and 4(d). A pixel is then classified as a ridge if it has only one neighboring ridge pixel in the window, and classified as a bifurcation if it has three neighboring ridge pixels. For display purpose, the ridge is marked by a red window square and bifurcation is marked by a blue square window.

When the fingerprint images are experimented, we reached a conclusion that the enhancement technique preserves bifurcation content. However the broken ridges cannot be preserved. The experimental results obtained are tabulated to evaluate the preservation of minutiae content in various fingerprint images. Figure 5 shows the bifurcation content in each fingerprint images after decompression and enhancement. Table I shows the performance values of the proposed method.

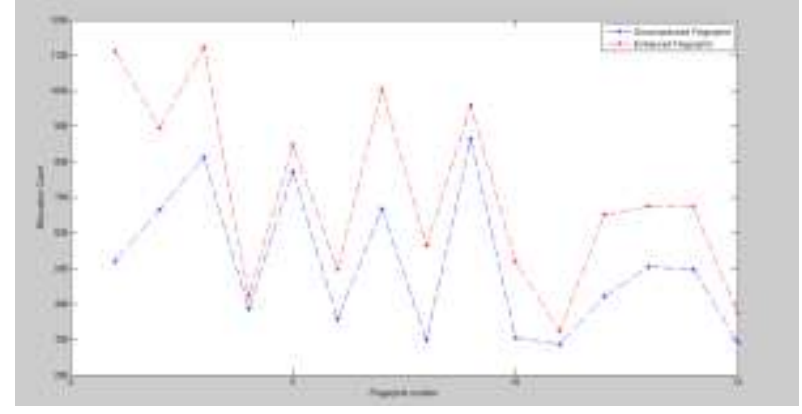

Figure 5. Performance Analysis

Table I

Experimental Results for Fingerprint Images

\begin{tabular}{|c|c|c|c|c|}
\hline \multirow{2}{*}{ Fingerprint Image } & \multicolumn{2}{|c|}{ After Decompression } & \multicolumn{2}{c|}{ After Enhancement } \\
\cline { 2 - 5 } & Ridge & Bifurcation & Ridge & Bifurcation \\
\hline 1.bmp & 554 & 521 & 510 & 1712 \\
\hline 2.bmp & 338 & 666 & 276 & 895 \\
\hline 3.bmp & 411 & 814 & 232 & 1122 \\
\hline 4. bmp & 650 & 385 & 426 & 423 \\
\hline 5.bmp & 543 & 772 & 427 & 848 \\
\hline 6.bmp & 548 & 358 & 410 & 497 \\
\hline 7. bmp & 571 & 668 & 413 & 1003 \\
\hline 8.bmp & 622 & 299 & 425 & 365 \\
\hline 9.bmp & 146 & 864 & 85 & 960 \\
\hline 10.bmp & 130 & 305 & 147 & 519 \\
\hline 11.bmp & 86 & 287 & 84 & 325 \\
\hline 12.bmp & 33 & 422 & 22 & 650 \\
\hline 13.bmp & 680 & 504 & 581 & 676 \\
\hline 14.bmp & 214 & 498 & 203 & 673 \\
\hline 15.bmp & 177 & 291 & 168 & 373 \\
\hline
\end{tabular}

\section{CONCLUSION}

In this paper, we propose an enhancement technique that is applied on compressed fingerprint images to make it better of fingerprint matching and so on. When the compressed fingerprint is decompressed, the image preserve only very less minutiae due to the noise in the image. So we applied Wiener2 filter to overcome the noise and thus to preserve more minutiae. Experimental results show that the enhancement technique preserves bifurcation content. However the broken ridges cannot be preserved. The method can be further improved by preserving ridges also. Latent fingerprints that are obtained from crime scenes can also consider for future works.

\section{ACKNOWLEDGMENTS}

Our thanks to all of them who had helped during this work. 


\section{REFERENCES}

[1] D. Maltoni, D. Miao, A. K. Jain, and S. Prabhakar, "Handbook of Fingerprint Recognition," 2nd ed. London, U.K.: Springer-Verlag, 2009.

[2] W. Pennebaker and J. Mitchell, "JPEG-Still Image Compression Standard," New York, NY, USA: Van Nostrand Reinhold, 1993.

[3] A. Skodras, C. Christopoulos, and T. Ebrahimi, "The JPEG 2000 still image compression standard," IEEE Signal Process. Mag., vol. 11, no. 5, pp. 36-58, Sep. 2001.

[4] T. Hopper, C. Brislawn, and J. Bradley," WSQ grayscale fingerprint image compression specification," Federal Bureau of Investigation, Criminal Justice Information Services, Washington, DC, USA, Tech. Rep. IAFIS-IC-0110V2, Feb. 1993.

[5] Jinshan Tang and Xiaoming Liu, “An Improved WSQ Fingerprint Image Compression Algorithm," IEEE Conference, 2008.

[6] Abdul Adeel Mohammed, Rashid Minhas, Q.M. Jonathan Wu, Maher A. Sid-Ahmed, “A Generic Fingerprint Image Compression Technique Based On Wave Atoms Decomposition," IEEE Conference 2009.

[7] S. Esakkirajan, T. Veerakumar, V. Senthil Murugan and R. Sudhakar, "Fingerprint Compression Using Contourlet Transform and Multistage Vector Quantization," International Journal of Computer, Information, Systems and Control Engineering, 2007.
[8] Shenqiu Zhang, and Cecilia Moloney, "Gray-Scale Fingerprint Image Compression Based on the Hybrid-NRCT," IEEE Conference 2009.

[9] M. Aharon, M. Elad, and A. M. Bruckstein, “'On the uniqueness of overcomplete dictionaries, and a practical way to retrieve them," J. Linear Algebra Appl., vol. 416, no. 1, pp. 48-67, 2006.

[10] M. Aharon, M. Elad, and A. M. Bruckstein, “The KSVD: An algorithm for designing of overcomplete dictionaries for sparse representation," IEEE Trans. Signal Process., vol. 54, pp. 4311-4322, 2006.

[11] S. Mallat and Z. Zhang, "Matching pursuits with timefrequency dictionaries," IEEE Trans. Signal Process., vol. 41, no. 12, pp. 3397-3415, Dec. 1993.

[12] Guangqi Shao, Yanping Wu, Yong A, Xiao Liu, and Tiande Guo, "Fingerprint Compression Based on Sparse Representation," IEEE Transactions on Image processing, Vol. 23, No. 2, February 2014.

[13] (2013, Jun.) Fingerprint Images [Online]. Available:http://pan.baidu.com/share/link?shareid= 3872475108 \& uk=2301599420 